\title{
OPEN Use of multiple metrics to assess antibiotic use in Italian children's hospitals
}

\author{
Carmen D'Amore ${ }^{1}$, Marta Luisa Ciofi degli Atti ${ }^{1 凶}$, Carla Zotti $^{2}$, Rosa Prato ${ }^{3}$, \\ Giuliano Guareschi ${ }^{4}$, Raffaele Spiazzi ${ }^{5}$, Gaetano Petitti ${ }^{6}$, Maria Luisa Moro ${ }^{7}$ \& \\ Massimiliano Raponi ${ }^{8}$
}

Quantification of antibiotic utilization is an essential component of antibiotic stewardship programs. In this multicentric study, we used different metrics to evaluate inpatient antibiotic use in children. The study objectives were to describe point prevalence of antibiotic use by indication and patient characteristics, to evaluate DOTs, LOTs and PDDs, and to compare PDDs to DDDs, which assume average maintenance dose per day in adults. All children hospitalized on the days of the study were included. Trained personnel collected demographic and clinical data from patients' clinical records. We recorded information about antibiotics administered on the date of data collection, and in the previous 30 days of hospitalization. Of 810 patients, 380 (46.9\%; Cl 95\%: 43.4-50.4) received one or more antibiotics; prevalence of use was $27.0 \%$ for prophylaxis $(219 / 810)$, and $20.7 \%(168 / 810)$ for treatment. Overall, 587 drugs were issued to the 380 patients receiving antibiotics ( 1.5 antibiotic per patient). When considering treatments, DOT and LOT per 100 patient-days were 30.5 and 19.1, respectively, resulting in a DOT/LOT ratio of 1.6. PDDs increased with age and approached DDDs only in children aged $\geq 10$ years; the ratio between PDDs estimated in children aged $\geq 10$ years and in 0-11 month-old infants ranged from 2 for sulfamethoxazole and trimethoprim, to 25 for meropenem. Our results confirm that DOT, LOT and PDD are better alternatives to DDD in children.

Improving the use of antibiotics is an important patient safety and public health issue, as well as a global priority ${ }^{1}$. The misuse of antibiotics, in fact, has contributed to the growing problem of antibiotic resistance, which has become one of the most serious and growing public health threats ${ }^{1,2}$. Children represent an excellent population for the selection of resistant bacterial pathogens after recent antimicrobial use $\mathrm{e}^{3,4}$. Therefore, the investigation of antibiotic consumption in the pediatric population is crucial, especially in the hospital setting where the risk of the emergence and transmission of bacterial resistance is dramatically increased ${ }^{3}$.

A growing body of evidence demonstrates that hospital based antibiotic stewardship programs (ASPs) can both optimize the treatment of infections and reduce adverse events associated with antibiotic use ${ }^{5-7}$. Quantification of antibiotic utilization is an integral part of ASPs. Hospital antibiotic consumption is commonly measured by calculating the daily defined doses (DDDs), as defined by the World Health Organization Collaborating Center for Drug Statistics Methodology, divided by a denominator indicating clinical activity (e.g. DDDs per 100 patient-days) ${ }^{8,9}$. DDD is calculated as the assumed average maintenance dose per day for a drug used for its main indication in adults. Even if DDDs allow the comparison of drug use between different settings and between different drugs, they do not reflect the dosage actually used for individual patients or specific patient populations ${ }^{10}$, and their use is not recommended in children ${ }^{11}$. To overcome these issues, in-hospital antibiotic use in children is frequently estimated by conducting point-prevalence surveys (PPS). Although the PPS design has been extensively used to evaluate antibiotic use, it cannot capture treatment duration. Other antibiotic consumption indicators are days of therapy (DOTs), length of therapy (LOTs) and prescribed daily doses (PDDs). DOTs are computed summing up the duration (in days) of each antibacterial drug received for treatments. LOTs

\footnotetext{
${ }^{1}$ Clinical Pathways and Epidemiology Unit, Bambino Gesù Children's Hospital, Rome, Italy. ${ }^{2}$ Department of Public Health and Pediatrics, University of Turin, Turin, Italy. ${ }^{3}$ Department of Medical and Surgical Sciences, University of Foggia, Ospedale "D’Avanzo" Policlinico Riuniti, Foggia, Italy. ${ }^{4}$ Ospedale Infantile Regina Margherita - AOU Città Della Salute E Della Scienza Di Torino, Torino, Italy. ${ }^{5}$ Ospedale dei Bambini - ASST Degli Spedali Civili di Brescia, Brescia, Italy. ${ }^{6}$ Azienda Ospedaliero Universitaria Consorziale Policlinico di Bari Ospedale Pediatrico "Giovanni XXIII", Bari, Italy. ${ }^{7}$ Regional Health and Social Agency, Emilia-Romagna Region, Italy. ${ }^{8}$ Medical Direction, Bambino Gesù Children's Hospital, Rome, Italy. ${ }^{\boxplus}$ email: marta.ciofidegliatti@opbg.net
} 


\begin{tabular}{|c|c|c|c|c|}
\hline & N. patients with at least 1 prescription & N. total patients & Prevalence \% (CI 95\%) & $P$ value \\
\hline \multicolumn{5}{|l|}{ Sex } \\
\hline Female & 162 & 379 & $50.9(46.1-55.8)$ & 0.02 \\
\hline Male & 215 & 422 & $42.7(37.7-47.9)$ & \\
\hline Missing & 3 & 9 & - & \\
\hline \multicolumn{5}{|l|}{ Age } \\
\hline 0-11 months & 47 & 132 & $35.6(27.5-44.4)$ & $<0.001$ \\
\hline 12-23 months & 40 & 123 & $32.5(24.4-41.6)$ & \\
\hline $2-4$ years & 100 & 182 & $54.9(47.4-62.3)$ & \\
\hline $5-9$ years & 74 & 139 & $53.2(44.6-61.7)$ & \\
\hline$\geq 10$ years & 119 & 233 & $51.1(44.5-57.7)$ & \\
\hline Missing & 1 & 1 & - & \\
\hline \multicolumn{5}{|c|}{ Length of hospital stay (days) } \\
\hline$\leq 7$ & 211 & 458 & $46.1(41.4-50.8)$ & 0.5 \\
\hline $08-30$ & 108 & 215 & $50.2(43.4-57.1)$ & \\
\hline$>30$ & 61 & 137 & $44.5(36.0-53.3)$ & \\
\hline \multicolumn{5}{|l|}{ Ward } \\
\hline Medical & 196 & 450 & $43.6(38.9-48.3)$ & 0.1 \\
\hline Surgical & 133 & 261 & $50.9(44.7-57.2)$ & \\
\hline Intensive care & 51 & 99 & $51.5(41.3-61.7)$ & \\
\hline \multicolumn{5}{|c|}{ Surgical procedure during present hospitalization } \\
\hline Yes & 148 & 231 & $64.1(57.5-70.3)$ & $<0.001$ \\
\hline No & 226 & 563 & $40.1(36.1-44.3)$ & \\
\hline Missing & 6 & 16 & - & \\
\hline \multicolumn{5}{|l|}{ Comorbidities } \\
\hline Yes & 128 & 306 & $41.8(36.2-47.6)$ & \\
\hline No & 242 & 482 & $50.2(45.7-54.8)$ & 0.02 \\
\hline Missing & 10 & 22 & - & \\
\hline \multicolumn{5}{|c|}{ Participating centre } \\
\hline 1 & 237 & 460 & $51.5(46.8-56.2)$ & $<0.001$ \\
\hline 2 & 52 & 154 & $33.8(26.4-41.8)$ & \\
\hline 3 & 23 & 35 & $65.7(47.8-80.9)$ & \\
\hline 4 & 68 & 161 & $42.2(34.5-50.3)$ & \\
\hline Total & 380 & 810 & $46.9(43.4-50.4)$ & \\
\hline
\end{tabular}

Table 1. Characteristics of patients receiving therapeutic and prophylactic antimicrobials in Italian Pediatric Hospitals, 2016.

are the number of days that a patient receives an antibacterial drug irrespective of the number of different drugs. DOTs and LOTs give an accurate estimation of polydrug therapy and duration of therapy, respectively; since they do not consider whether a nonstandard dose was given ${ }^{12}$, they could be considered as accurate metrics of drug use for children. The PDD provides the average daily amount of a drug that is actually prescribed ${ }^{13}$. In this multicentric study, we adopted different metrics to evaluate inpatient antibiotic use in hospitalized children. The study objectives were to describe point prevalence of antibiotic use by indication and patient characteristics, to estimate DOTs, LOTs and PDDs as additional metrics to quantify antibiotic use, and to compare PDDs to DDDs by antibiotic and age group.

\section{Results}

Point prevalence of antibiotic use. In our study, we included 810 children. Patients were mostly males $(\mathrm{n}=422,52 \%)$, aged $\geq 10$ years years $(\mathrm{n}=233,29 \%)$ and with a length of hospital stay $\leq 7$ days $(\mathrm{n}=458,57 \%)$ (Table 1). About $38 \%$ of the patients (306/810) had at least one chronic disease; the most frequent chronic diseases were congenital heart diseases $(70 / 306 ; 23 \%)$, genetic syndromes $(38 / 306 ; 12 \%)$, chronic respiratory diseases (22/306; 7\%), neuromuscular (19/306; 6\%), and chronic renal diseases (18/306; 6\%). Two hundred and thirty-one patients $(29 \%)$ underwent at least one surgical procedure during hospitalization, for a total of 331 surgical procedures. The majority of patients (450/810; 56\%), were admitted to medical units, $32 \%(261 / 810)$ to surgical units and $12 \%(99 / 810)$ to intensive care units (Table 1$)$.

Of the 810 patients, 380 (46.9\%; CI 95\%: 43.4-50.4) received one or more antibiotics. The proportion of patients receiving antibiotics significantly varied by hospital, age, sex, comorbidities and surgical procedures. In particular, the prevalence of use was significantly higher in patients aged $\geq 2$ years compared to those in the first 


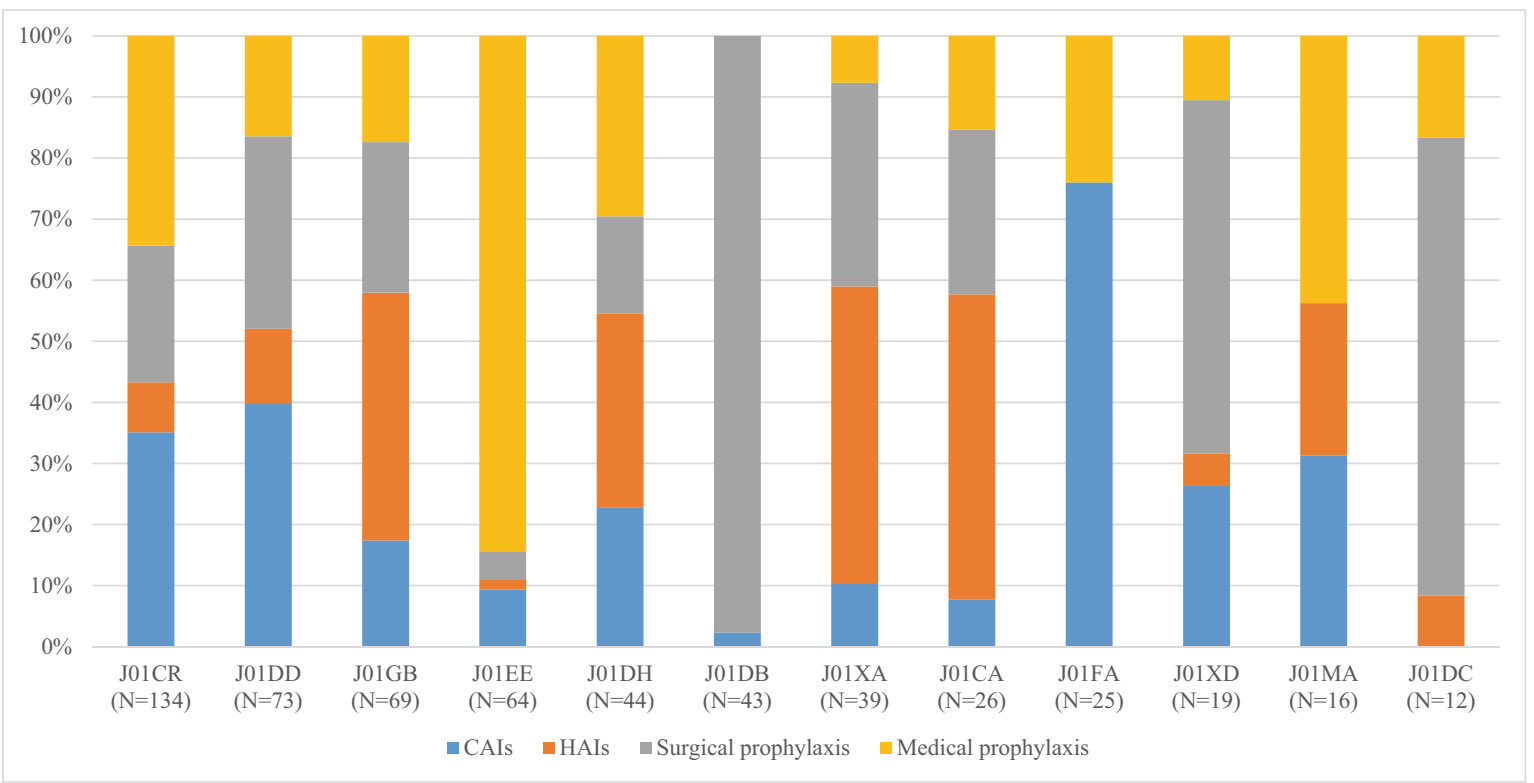

Figure 1. Proportions of treatment by antibiotic drugs and indication of use. Only antibiotic drugs with a frequency of treatment $>1.0 \%$ are showed. J01CR: Combinations of penicillins, incl. beta-lactamase inhibitors; J01DD: third generation cephalosporins; J01GB: Other aminoglycosides; J01EE: Combinations of sulfonamides and trimethoprim; J01DH: Carbapenems; incl. derivatives; J01DB: First-generation cephalosporins; J01XA: Glycopeptide antibacterials; J01CA: Penicillins with extended spectrum; J01FA: Macrolides; J01XD: Imidazole derivatives; J01MA: Fluoroquinolones; J01DC: Second-generation cephalosporins.

year of life, in males with respect to females, in patients without comorbidities compared to those who had at least one chronic disease, and in patients who underwent at least one surgical procedure (Table 1).

Prophylaxis was the main indication for antibiotic use $(217 / 380 ; 57.1 \%)$. Children receiving antibiotics for surgical or medical prophylaxis accounted for $14.3 \%(116 / 810)$ and $12.7 \%(103 / 810)$ of the hospitalized patients, respectively. Surgical prophylaxis lasted more than $24 \mathrm{~h}$ in $76.7 \%$ of the patients $(89 / 116)$. Patients treated for CAIs were $13.2 \%$ (107/810) of children, followed by patients treated for HAIs $(61 / 810 ; 7.5 \%)$.

Overall, 587 drugs were issued to the 380 patients receiving antibiotics ( 1.5 antibiotic per patient), mainly administered intravenously (465/587; 79.2\%). Prescriptions for treatments were $42.8 \%$ (251/587); of those, the majority of antibiotics was administered for CAIs (147/251; 58.6\%), and the remaining for HAIs $(104 / 251 ; 41.4 \%)$. Overall, microbiological cultures were performed before starting the antibiotic therapy in $54.5 \%$ of the treatments (137/251). In $23.1 \%$ of the prescriptions (58/251), the results of the antibiogram were available and the antibiotic therapy could be considered as targeted, whereas $68.1 \%(171 / 251)$ of the treatments were empirical. The main infection sites were respiratory tract infections (both low tract and pneumonia), bloodstream infections, and urinary tract infections, which were reported as the reason for therapy in respectively $29.9 \%, 23.5 \%$ and $2.4 \%$ of the antibiotic treatments for infections.

The top three antibiotic classes were combinations of penicillins including $\beta$-lactamase inhibitors (J01CR; 134/587, 22.8\%), third-generation cephalosporins (J01DD; 73/587, 12.4\%) and aminoglycosides (J01GB; 69/587, $11.8 \%$, Fig. 1).

The pattern of antibiotic use varied by indication: about $98.0 \%(42 / 43)$ of first-generation cephalosporin prescriptions (J01DB) were issued for surgical prophylaxis, followed by second-generation cephalosporins (J01DC; 9/12, 75.0\%) and imidazole derivatives (J01XD; 11/19, 57.9\%) (Fig. 1).

Combinations of sulphonamides and trimethoprim (J01EE), fluoroquinolones (J01MA) and combinations of penicillins including $\beta$-lactamase inhibitors (J01CR) were mainly prescribed for medical prophylaxis $(84.4 \%$, $43.8 \%$ and $34.3 \%$ of the treatments, respectively) (Fig. 1 ).

CAIs represented the main indication of use for macrolides (J01FA; 19/25, 76.0\%), third generation cephalosporins (J01DD; 29/73, 39.7\%) and combinations of penicillins including $\beta$-lactamase inhibitors (J01CR; 47/134, $35.1 \%$ ) whereas HAIs were mainly reported for penicillins with extended spectrum (J01CA; 13/26, 50.0\%), glycopeptides (J01XA; 19/39, 48.7\%) and aminoglycosides (J01GB; 28/69, 40.6\%).

Estimation of DOTs and LOTs. DOTs and LOTs were estimated considering 414 inpatient antibiotic treatments, over a total of 9458 inpatient days, retrospectively evaluated considering the 30 days prior to the collection of the point prevalence data on antibiotic use. Overall, we estimated 30.5 DOTs per 100 patient days and a 19.1 LOT per 100 patient days resulting in a DOT/LOT ratio of 1.6 (Table 2). DOT and LOT/100 inpatient days varied significantly by hospital, age, sex, type of ward, and comorbidities. In detail, antibiotic use was higher in males with respect to females, in children aged $<10$ years with respect to those aged $\geq 10$ years and in the ICUs with respect to medical and surgical wards (Table 2). DOT/100 patient days by type of drug confirmed that penicillins, aminoglycosides, carbapenems and cephalosporins were the antibiotics most frequently prescribed 


\begin{tabular}{|c|c|c|c|c|c|c|}
\hline & $\begin{array}{l}\text { Number of patient } \\
\text { days }\end{array}$ & DOT/100 patient days & $P$ value $_{\text {DOT }}$ & LOT/100 patient days & $P_{\text {value }_{\text {LOT }}}$ & DOT/LOT ratio \\
\hline \multicolumn{7}{|c|}{ 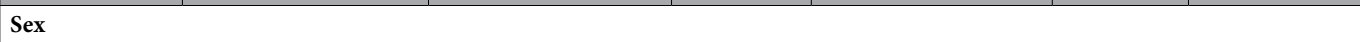 } \\
\hline Male & 4779 & 33.9 & $<0.001$ & 20.7 & $<0.001$ & 1.6 \\
\hline Female & 4653 & 27.8 & & 17.8 & & 1.6 \\
\hline \multicolumn{7}{|l|}{ Age classes } \\
\hline $0-11$ months & 1496 & 30.1 & $<0.001$ & 19.0 & $<0.001$ & 1.6 \\
\hline $12-23$ months & 2063 & 40.3 & & 24.8 & & 1.6 \\
\hline $2-4$ years & 1996 & 26.5 & & 18.4 & & 1.4 \\
\hline $5-9$ years & 1282 & 33.5 & & 18.5 & & 1.8 \\
\hline$\geq 10$ years & 2680 & 25.1 & & 15.7 & & 1.6 \\
\hline \multicolumn{7}{|l|}{ Ward } \\
\hline Medical & 5362 & 29.7 & $<0.001$ & 18.6 & $<0.001$ & 1.6 \\
\hline Surgical & 2450 & 20.6 & & 13.4 & & 1.5 \\
\hline Intensive Care & 1736 & 47.2 & & 28.5 & & 1.7 \\
\hline \multicolumn{7}{|c|}{ Length of hospital stay (days) } \\
\hline$\leq 7$ & 4154 & 30.5 & 0.7 & 17.9 & $<0.001$ & 1.7 \\
\hline $08-30$ & 1816 & 31.3 & & 22.0 & & 1.4 \\
\hline$>30$ & 3578 & 30.2 & & 18.8 & & 1.6 \\
\hline \multicolumn{7}{|l|}{ Comorbidities } \\
\hline Yes & 4343 & 27.3 & $<0.001$ & 20.1 & $<0.001$ & 1.3 \\
\hline No & 4884 & 33.6 & & 17.7 & & 1.9 \\
\hline \multicolumn{7}{|c|}{ Participating center } \\
\hline 1 & 5799 & 34.6 & $<0.001$ & 21.3 & $<0.001$ & 1.6 \\
\hline 2 & 1612 & 40.6 & & 24.9 & & 1.6 \\
\hline 3 & 251 & 31.1 & & 20.7 & & 1.5 \\
\hline 4 & 1886 & 9.3 & & 7.1 & & 1.3 \\
\hline Total & 9548 & 30.5 & & 19.1 & & 1.6 \\
\hline
\end{tabular}

Table 2. Patient days, DOT-LOT per 100 patient days and DOT/LOT ratio calculated for antibiotics provided for therapeutic purposes by sex, age classes, hospital ward, lenght of hospital stay, comorbidities and participating center.

for treatment, with estimated DOT/100 patient days of 7, 6 and 4 (Fig. 2). Targeted therapies had lower DOT/100 patient days than empirical therapies (8.0 DOT/100 vs. $21.0 \mathrm{DOT} / 100)$.

The overall DOT/LOT ratio of 1.6 indicated that the combination antibacterial treatment was common (Table 2).

Estimation of PDDs and comparison with DDDs. Table 3 shows the median PDDs by age groups for the eight most frequently prescribed treatments. The PDDs increased with age: the ratio between PDDs estimated in children aged $\geq 10$ years and PDDs in children aged $0-11$ months did greatly vary by antibiotic, and ranged from a minimum of 2 for sulfamethoxazole and trimethoprim, to a maximum of 25 for meropenem. The PDD approached the DDD only in children aged $\geq 10$ years: in this age group the PDDs were equal to the DDDs for gentamicin and ceftriaxone, while the median PDD was lower than the DDD for piperacillin/amoxicillin and beta-lactamase inhibitors, sulfamethoxazole and trimethoprim, amikacin and cefazolin. The PDD was higher than the DDD only for meropenem.

\section{Discussion}

Out of more than 800 children included in our study, $47 \%$ were treated with at least one antibiotic. This estimate is higher than reported in European hospitalized children, where the point prevalence of antibiotic use was $39.6 \%{ }^{14}$. We showed how hospital and patient characteristics, such as age and comorbidities, are related to the use of antibiotics, and other authors have shown that the prevalence of antibiotic use is higher in third-level hospitals where patients generally have more complex conditions ${ }^{15}$. As patient population characteristics are major determinants of antibiotic consumption, risk adjustment should be adopted to compare antibiotic consumption data among hospitals and over-time. In our results, previous surgery was significantly associated with antibiotic use and surgical prophylaxis was the most frequent indication. As previously documented ${ }^{14-16}$, we showed a suboptimal appropriateness of antibiotic surgical prophylaxis; in fact, there was still a proportion of patients treated with non-recommended broad-spectrum active substances, such as III-IV generation cephalosporins.

Another worrying aspect is the duration of surgical prophylaxis; in fact, although a prolonged exposure does not reduce the risk of surgical site infections, it represents a relevant ecological risk ${ }^{17}$. In our study, approximately $77 \%$ of the patients undergoing surgical prophylaxis received surgical prophylaxis for more than $24 \mathrm{~h}$. This is in 


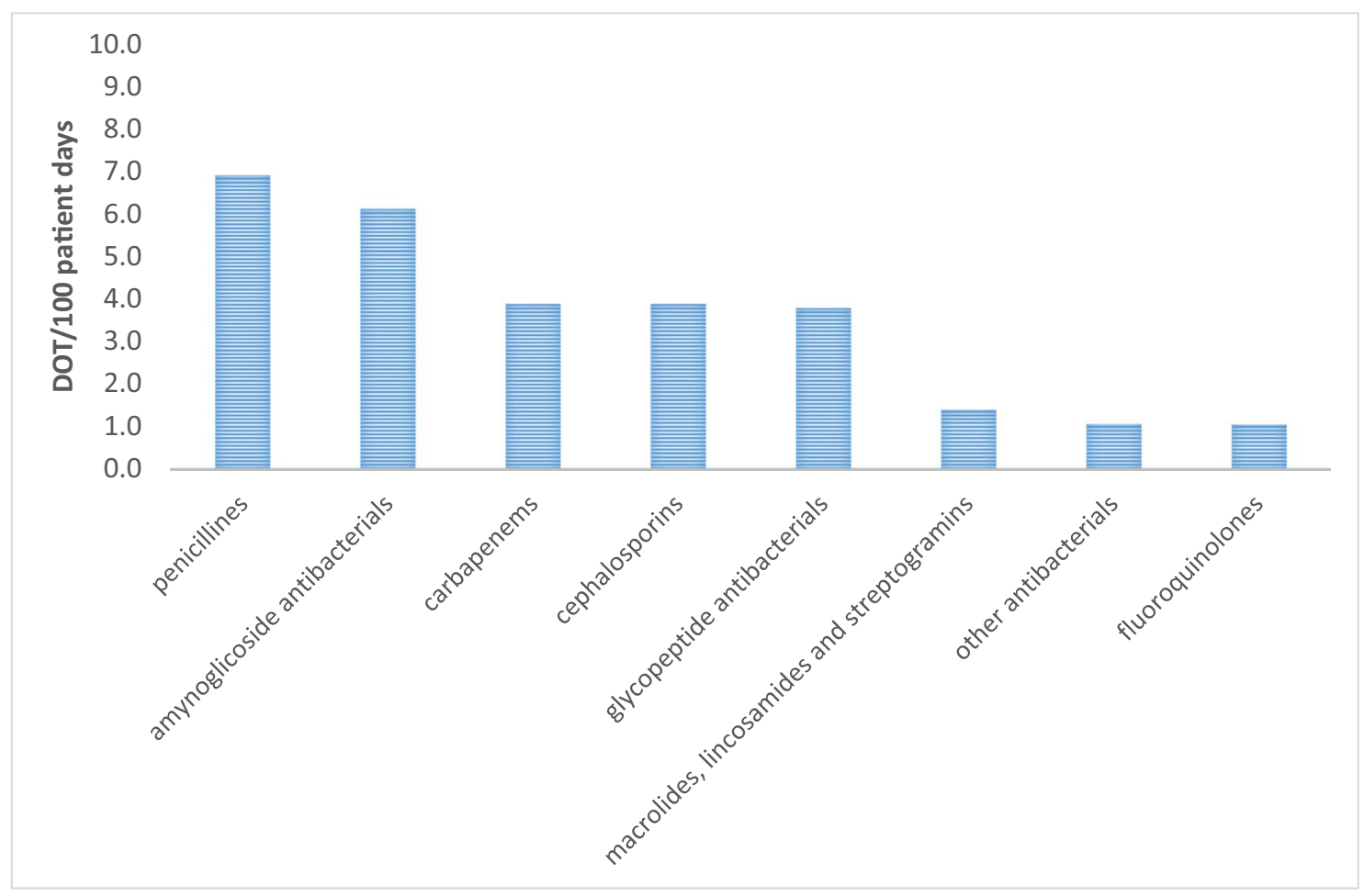

Figure 2. DOT/100 patient days by antibiotic drugs provided for therapeutic purposes. Only antibiotic drugs with more than $1 \mathrm{DOT} / 100$ patient days are shown in the graphic.

\begin{tabular}{|c|c|c|c|c|c|c|c|}
\hline \multirow[b]{2}{*}{ Antibiotic molecules } & \multicolumn{7}{|c|}{ Median PDDs (g) by age classes } \\
\hline & No. of therapies & DDD (g) & 0-11 Months (IQR) & 12-23 Months (IQR) & 2-4 Years (IQR) & 5-9 Years (IQR) & $\geq 10$ Years $(\mathrm{IQR})$ \\
\hline $\begin{array}{l}\text { Piperacillin and beta-lactamase inhibi- } \\
\text { tors }\end{array}$ & 78 & 14.0 & $0.3(0.07-0.6)$ & $1.0(0.8-1.6)$ & $3.0(2.7-4.5)$ & $7.0(6.0-9.0)$ & $12.0(9.0-12.0)$ \\
\hline Meropenem & 71 & 2.0 & $0.1(0.06-0.1)$ & $0.3(0.2-0.4)$ & $0.8(0.6-1.3)$ & $1.8(1.1-1.9)$ & $3.0(2.1-4.5)$ \\
\hline $\begin{array}{l}\text { Amoxicillin and beta-lactamase } \\
\text { inhibitors }\end{array}$ & 72 & 3.0 & $0.3(0.3-0.3)$ & $0.7(0.4-0.8)$ & $0.7(0.4-1.2)$ & $1.5(1.0-1.8)$ & $2.0(1.7-2.0)$ \\
\hline Sulfamethoxazole and trimethoprim & 70 & 1.9 & $0.01(0.01-0.01)$ & $0.3(0.04-0.4)$ & $0.1(0.05-0.3)$ & $0.2(0.09-0.5)$ & $0.3(0.2-0.3)$ \\
\hline Amikacin & 64 & 1.0 & $0.02(0.02-0.03)$ & $0.06(0.05-0.07)$ & $0.2(0.2-0.6)$ & $0.4(0.3-0.4)$ & $0.7(0.5-1.0)$ \\
\hline Ceftriaxone & 63 & 2.0 & $0.3(0.3-0.3)$ & $0.4(0.4-0.7)$ & $1.0(0.5-1.0)$ & $1.5(1.0-2.0)$ & $2.0(1.0-2.0)$ \\
\hline Cefazolin & 61 & 3.0 & $0.1(0.05-0.2)$ & $0.3(0.2-0.3)$ & $0.7(0.5-1.0)$ & $1.3(0.6-1.8)$ & $2.0(2.0-3.0)$ \\
\hline Gentamicin & 55 & 0.2 & $0.01(0.004-0.01)$ & $0.01(0.01-0.02)$ & $0.05(0.04-0.06)$ & $0.1(0.08-0.1)$ & $0.2(0.1-0.3)$ \\
\hline
\end{tabular}

Table 3. Median PDDs of the most used antibiotic molecules provided for therapeutic purposes by age classes.

line with previous prevalence surveys, which estimated a proportion of children subject to prolonged surgical prophylaxis (>1 day) fluctuating between 67 and $78 \%$ in Europe $e^{3,16}$. These data show that even in the most documented evidence-based area of practice, which recommends a surgical antimicrobial prophylaxis duration of less than $24 \mathrm{~h}$, clinicians tend to overprescribe, and this is a global phenomenon not exclusively limited to Europe ${ }^{3}$. Combinations of sulfonamides and trimethoprim and combinations of penicillins including beta-lactamase inhibitors have been the most widely used active substances for medical prophylaxis, being widely used to prevent opportunistic infections in neutropenic patients ${ }^{18}$. Medical prophylaxis, as well as infection treatments, include a wide range of diseases and it is therefore difficult to identify unambiguous recommendations in patients with very different clinical conditions.

Although point prevalence surveys are useful to assess the pattern of in-hospital antibiotic prescriptions, antibiotic use should be preferably expressed with multiple metrics ${ }^{19}$. We collected DOT, LOT and PDDs as more specific antibiotic indicators for infection treatments with respect to DDDs, which are not recommended for children because doses used are typically much smaller than in adult populations, so this measure underestimates patient exposure to drugs ${ }^{11}$. DOT and LOT have been used to monitor antibiotic use and to assess the impact of antibiotic stewardship programs on improving appropriateness of prescriptions in children ${ }^{12,13}$. We showed that calculation of DOT and LOT by retrospectively reviewing medical records was feasible. 
DOT/100 patient days was comparable with antibiotic prescriptions for infections in a secondary pediatric hospital in the Netherlands, where the average DOT/100 in neonatal and pediatric wards was between 23 and $45^{20}$. Intensive care units were the setting with the highest antibiotic use, with LOT and DOT rates per 100 days of 28.5 and 47.2, respectively. Moreover, a DOT/LOT ratio of 1.6 confirms the frequent resort to multi-therapies found in the prevalence survey where an equal average number of active substances per patient was detected.

Both LOTs and DOTs included the underlying assumption that antibiotic dosing was appropriate. PDDs represented the real prescribed doses of drugs in children. As reported also in a previous study ${ }^{3}$, our study showed that PDD increased with age and approached to DDD only in children aged $\geq 10$ years. Although there was no difference between DDDs and PDDs of antibiotic drugs belonging to the class of aminoglycosides and cephalosporins in this age group, a difference was observed for other antibiotic drugs. PDDs lower than DDDs could be explained considering that children's weight is lower than the standard weight used to compute DDDs, otherwise PDDs higher than DDDs could be reconducted to the patients' clinical conditions to be treated. It could be the case of meropenem that is frequently used to treat serious healthcare associated bloodstream infections requiring more aggressive dosages.

This multicentre study was conducted in four stand-alone tertiary care children's hospitals located in Regions in the North, Centre, and South of Italy. Trained investigators collected data regarding antibiotic prescriptions from clinical records minimising the effect of information bias on our study results. Additionally, in contrast to studies that are based on information collected directly from patients medical clinical records, we had no need to restrict the sample size. The study limitations are inherent to the epidemiological methods of our cross-sectional survey, where the main purpose was to describe prescribing patterns in hospitals. The overall rate provided is the average. We did not control for patient case mix, disease incidence, prevalence of different types of infections, variations in resistance levels, institutional factors, all of which can influence antibiotic use patterns ${ }^{21}$.

In conclusion, our results confirm that DOT, LOT and PDD are better alternatives to DDD in children. The availability of electronic clinical records can greatly enhance data accessibility to calculate DOT, LOT and PDD data and use those data within antibiotic stewardship programs, as alternative to DDDs in pediatric population.

\section{Methods}

Study design and setting. This study was conducted between November and December 2016 and involved four tertiary care children's hospitals in Italy: Ospedale Infantile Regina Margherita (Turin, in the Region of Piedmont), Ospedale dei Bambini di Brescia (Brescia, in the Region of Lombardy), Bambino Gesù Children's Hospital (Rome, in the Region of Lazio) and Ospedale Pediatrico Giovanni XXIII (Bari, in the Region of Apulia). These tertiary care children's hospitals have a total number of beds ranging from a minimum of 157 to a maximum of 607 , with an annual number of inpatients ranging from 5700 to 27,000 . Pediatric surgeries and pediatric intensive care units are present in all the participating centres; Onco-Haematological Units and Neonatal Intensive Care Units are present in three centres.

Data collection. The data collection was carried out during a calendar week and involved all children hospitalized on the study days. Data were collected by trained personnel from patients' paper medical charts. Information collected for each patient included: age, sex, length of hospital stay, and ward type. To assess point prevalence, we collected information about antibiotics administered during the days of data collection. To estimate DOT and LOT, we retrospectively recorded information about antibiotics administered during hospitalization, up to 30 inpatient days prior to the week of data collection. Information on antibiotics included Anatomical Therapeutic Chemical Classification code, ATC J01) ${ }^{14}$, drug brand name, route of administration, dosage, number of doses per day, and indication for antibiotic administration, defined as community-acquired infections (CAIs), hospital-acquired infections (HAIs), medical or surgical prophylaxis. For antibiotics used for treating infections, collected information included also the site of infection and whether microbiological investigations were performed prior to starting antibiotic therapy. The protocol of this study was approved by the Ethical Committee of the Bambino Gesù Children's Hospital (N 1238_OPBG_2016) and all methods were performed following relevant guidelines and regulations. Since data were collected by reviewing medical charts and were analyzed anonymously, informed consent was not deemed necessary. The need for informed consent was waived by the Ethical Committee of the Bambino Gesù Children's Hospital.

All the collected data were uploaded on REDCap (Research Electronic Data Capture) database which is a secure web application for building and managing online surveys and databases, available at no charge to notfor-profit institutions ${ }^{22}$. All the data were analyzed anonymously.

Statistical analysis. Patients were described according to demographic and clinical factors. Collected data were presented as count and proportions (categorical data) or median and interquartile range (IQR, continuous data). The wards where the patients were hospitalized were categorized as medical (e.g. general neonatal and pediatric wards, oncology and hematology), surgical (e.g. neonatal surgery, pediatric surgery, cardiac surgery, neurosurgery, ENT, orthopedics) and intensive care units (ICUs) (e.g. pediatric intensive care unit, neonatal intensive care unit, and cardiac intensive care unit).

The metrics we calculated included point prevalence of antibiotic use, DOTs, LOTs and PDDs. The prevalence of antibiotic use was calculated as the ratio between the number of patients under treatment with at least one antibiotic on the survey day, and the total number of hospitalized patients. Prevalence of use was computed by age group (0-11 months, 12-23 months, 2-4 years; 5-9 years; $\geq 10$ years), sex, hospital ward, lenght of hospital stay, comorbidities, surgical procedures during hospitalization, participating centre and indication. Comparisons among groups were conducted using the Chi-squared test. 
Antibiotic drugs were classified according to the Anatomical Therapeutic Chemical Classification code (ATC: J01); a percentage distribution of antibiotic drugs substances by indication of use was computed considering the antibiotic prescription administered on the survey date and those received in the previous 30 days.

DOTs and LOTs were computed on treatments ${ }^{20}$, considering the 30 -days study period. For each patient, DOTs were computed summing up the duration (in days) of each antibacterial drug received for treating infections. LOTs were the number of days that a patient received an antibacterial drug irrespective of the number of different drugs ${ }^{12}$. DOTs/100 patient days and LOTs/100 patient days were computed stratifying for sex, age classes, ward type, type of active substances and targeted versus empirical therapy; groups comparisons were performed using Student's t-test and one way ANOVA test. PDDs were defined as the observed dose received by each patient in grams and was calculated for the most frequently used antibiotics. Due to the extremely large differences in dosing in pediatrics, ranging from pre-term neonates to teenagers, median PDDs, and the respective IQRs, were estimated and stratified into five age groups (1, 0-11 months; 2, 12-23 months; 3, 2-4 years; 4, $5-9$ years; $5, \geq 10$ years), as previously described ${ }^{3}$. All statistical analyses were conducted using STATA 13 (Stata Corporation, College Station, Texas, USA).

Received: 17 September 2020; Accepted: 25 January 2021

Published online: 11 February 2021

\section{References}

1. CDC. Core Elements of Hospital Antibiotic Stewardship Programs. Atlanta, GA: US Department of Health and Human Services, CDC; 2014. http://www.cdc.gov/getsmart/healthcare/implementation/core-elements.html.

2. Huttner, A. et al. Antimicrobial resistance: a global view from the 2013 World Healthcare-Associated Infections Forum. Antimicrob. Resist. Infect. Control. 2, 31 (2013).

3. Amadeo, B. et al. ESAC III hospital care subproject group. european surveillance of antibiotic consumption (ESAC) point prevalence survey 2008: paediatric antimicrobial prescribing in 32 hospitals of 21 European countries. J. AntimicrobChemother. 65, 2247-2252 (2010).

4. Chung, A. et al. Effect of antibiotic prescribing on antibiotic resistance in individual children in primary care: prospective cohort study. BMJ 335, 429 (2007).

5. CDC. Core Elements of Hospital Antibiotic Stewardship Programs. Atlanta, GA: US Department of Health and Human Services, CDC (2014). http://www.cdc.gov/getsmart/healthcare/implementation/core-elements.html.

6. Centers for Disease Control and Prevention. Get smart: know when antibiotics work. http://www.cdc.gov/getsmart/healthcare/ Accessed06/02/2020.

7. Davey, P. et al. Interventions to improve antibiotic prescribing practices for hospital inpatients. Cochrane Database Syst. Rev. 2, CD003543 (2017).

8. de With, K., Bestehorn, H., Steib-Bauert, M. \& Kern, W. V. Comparison of defined versus recommended versus prescribed daily doses for measuring hospital antibiotic consumption. Infection 37, 349-352 (2009).

9. Hutchinson, J. M. et al. Measurement of antibiotic consumption: a practical guide to the use of the Anatomical Thgerapeutic Chemical classification and Definied Daily Dose system methodology in Canada. Can. J. Infect. Dis. 15, 29-35 (2004).

10. Liem, T. B., Heerdink, E. R., Egberts, A. C. \& Rademaker, C. M. Quantifying antibiotic use in paediatrics: a proposal for neonatal DDDs. Eur. J. Clin. Microbiol. Infect. Dis. 29, 1301-1303 (2010).

11. WHO International Working Group for Drug Statistics Methodology, WHO Collaborating Centre for Drug Statistics Methodology, WHO Collaborating Centre for Drug Utilization Research and Clinical Pharmacological Services. Introduction to Drug Utilization Research (2013).

12. Schechner, V., Temkin, E., Harbarth, S., Carmeli, Y. \& Schwaber, M. J. Epidemiological interpretation of studies examining the effect of antibiotic usage on resistance. Clin. Microbiol. Rev. 26, 289-307 (2013).

13. WHO Collaborating Centre for Drug Statistics Methodology, Guidelines for ATC classification and DDD assignment 2016. Oslo (2016).

14. Versporten, A. et al. The antibiotic resistance and prescribing in European Children project: a neonatal and pediatric antimicrobial web-based point prevalence survey in 73 hospitals worldwide. Pediatr. Infect. Dis. J. 32, e242-e253 (2013).

15. Gharbi, M. et al. Using a simple point-prevalence survey to define appropriate antibiotic prescribing in hospitalised children across the UK. BMJ Open 6, e012675 (2016).

16. Harbarth, S. et al. Prolonged antibiotic prophylaxis after cardiovascular surgery and its effect on surgical site infections and antimicrobial resistance. Circulation 101, 2916-2921 (2000).

17. Versporten, A. et al. The Worldwide Antibiotic Resistance and Prescribing in European Children (ARPEC) point prevalence survey: developing hospital-quality indicators of antibiotic prescribing for children. J. AntimicrobChemother. 71, 1106-1117 (2016).

18. Cecinati, V. et al. Antibiotic prophylaxis in children with cancer or who have undergone hematopoietic cell transplantation. Eur. J. ClinMicrobiol. Infect. Dis. 33, 1-6 (2014).

19. StanicBenic, M. et al. Metrics for quantifying antibiotic use in the hospital setting: results from a systematic review and international multidisciplinary consensus procedure. J. AntimicrobChemother. 73(suppl_6), vi50-vi58. https://doi.org/10.1093/jac/dky11 8 (2018).

20. Quaak, C. H., Cové, E., Driessen, G. J. \& Tramper-Stranders, G. A. Trends in paediatric inpatient antibiotic therapy in a secondary care setting. Eur. J. Pediatr. 177, 1271-1278. https://doi.org/10.1007/s00431-018-3185-z (2018).

21. Versporten, A. et al. Antimicrobial consumption and resistance in adult hospital inpatients in 53 countries: results of an internetbased global point prevalence survey. Lancet Glob. Health 6, e619-e629. https://doi.org/10.1016/S2214-109X(18)30186-4 (2018).

22. Harris, P. A. et al. Research electronic data capture (REDCap) - a metadata-driven methodology and workflow process for providing translational research informatics support. J. Biomed. Inform. 42, 377-381 (2009).

\section{Author contributions}

C.D.A. prepared the study protocol and the data collection form, trained the hospital personnel to collect the data, conducted the study analysis and drafted the manuscript. M.C.D.A. conceived the study, participated in data analysis and interpretation, revised the manuscript and supervised the whole study. G.G., R.S., G.P. and M.R. coordinated the study and the data collection in participating hospitals. C.Z., R.P. and M.L.M. helped in the study conception and in data analysis and interpretation. All authors critically reviewed and approved the manuscript. 


\section{Funding}

This work was supported by the Centro per il Controllo e la Prevenzione delle Malattie (CCM) of the Ministry of Health within the Project "Buone pratiche per la sorveglianza e il controllo dell'antibioticoresistenza".

\section{Competing interests}

The authors declare no competing interests.

\section{Additional information}

Correspondence and requests for materials should be addressed to M.L.C.d.

Reprints and permissions information is available at www.nature.com/reprints.

Publisher's note Springer Nature remains neutral with regard to jurisdictional claims in published maps and institutional affiliations.

(c) (i) Open Access This article is licensed under a Creative Commons Attribution 4.0 International License, which permits use, sharing, adaptation, distribution and reproduction in any medium or format, as long as you give appropriate credit to the original author(s) and the source, provide a link to the Creative Commons licence, and indicate if changes were made. The images or other third party material in this article are included in the article's Creative Commons licence, unless indicated otherwise in a credit line to the material. If material is not included in the article's Creative Commons licence and your intended use is not permitted by statutory regulation or exceeds the permitted use, you will need to obtain permission directly from the copyright holder. To view a copy of this licence, visit http://creativecommons.org/licenses/by/4.0/.

(C) The Author(s) 2021 